\title{
The Oslo Cyclotron Laboratory
}

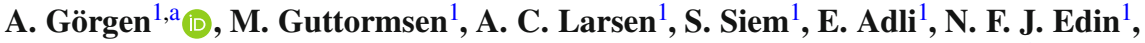 \\ H. Gjersdal ${ }^{1}$, G. Henriksen ${ }^{1,2,3}$, E. Malinen ${ }^{1,4}$, V. Modamio ${ }^{1}$, B. Schoultz ${ }^{5}$, \\ P. A. Sobas ${ }^{1}$, T. A. Theodossiou ${ }^{6}$, J. C. Wikne ${ }^{1}$ \\ ${ }^{1}$ Department of Physics, University of Oslo, Oslo, Norway \\ 2 Institute of Basic Medical Sciences, University of Oslo, Oslo, Norway \\ ${ }^{3}$ Norwegian Medical Cyclotron Centre, Sognsvannsveien 20, Oslo 0372, Norway \\ ${ }^{4}$ Department of Medical Physics, Oslo University Hospital, Oslo, Norway \\ ${ }_{6}^{5}$ Department of Chemistry, University of Oslo, Oslo, Norway \\ ${ }^{6}$ Department of Radiation Biology, Institute for Cancer Research, Radium Hospital, Oslo University Hospital, \\ Oslo, Norway
}

Received: 10 November 2020 / Accepted: 22 January 2021

(C) The Author(s) 2021

\begin{abstract}
Research at the Oslo Cyclotron Laboratory at the University of Oslo is focused on spectroscopy experiments for nuclear structure and nuclear astrophysics using the Oslo Scintillator Array OSCAR. Light-ion beams from the $K=35$ cyclotron are furthermore used for studies in radiation biology and medical physics, for research and development related to medical isotope production, and for irradiation of materials and electronics components. Here we present an overview of the laboratory and its research infrastructure, give a brief discussion of the respective research programs and methods, and present recent highlights.
\end{abstract}

\section{Introduction}

The Oslo Cyclotron Laboratory (OCL) is operated by the Department of Physics at the University of Oslo. It is located in the basement of the Physics Building on the Blindern Campus of the University. The laboratory is associated with the nuclear physics section of the Department of Physics and is supported by a technical staff of 2.5 FTE. The scientific program of OCL is led by academic staff from the Department of Physics and the University Hospital, but the laboratory has a wider user community of scientists from international institutions, who participate in collaborative experiments with local researchers. The scientific program spans a wide area of research topics such as nuclear structure and nuclear astrophysics, radiation biology, and medical applications of ion beams, as will be described in the following sections.

OCL welcomes proposals for experiments from external users. An internal committee composed of academic staff from the Department of Physics reviews proposals and allocates beam time. Because of the limited manpower resources at OCL, any experiment proposed by external users requires strong involvement from local scientists. The laboratory receives some base funding from the Department of Physics, but research projects are expected to contribute to the running costs of the laboratory through research grants. The cyclotron operates typically

\footnotetext{
a e-mail: andreas.gorgen@fys.uio.no (corresponding author)
} 

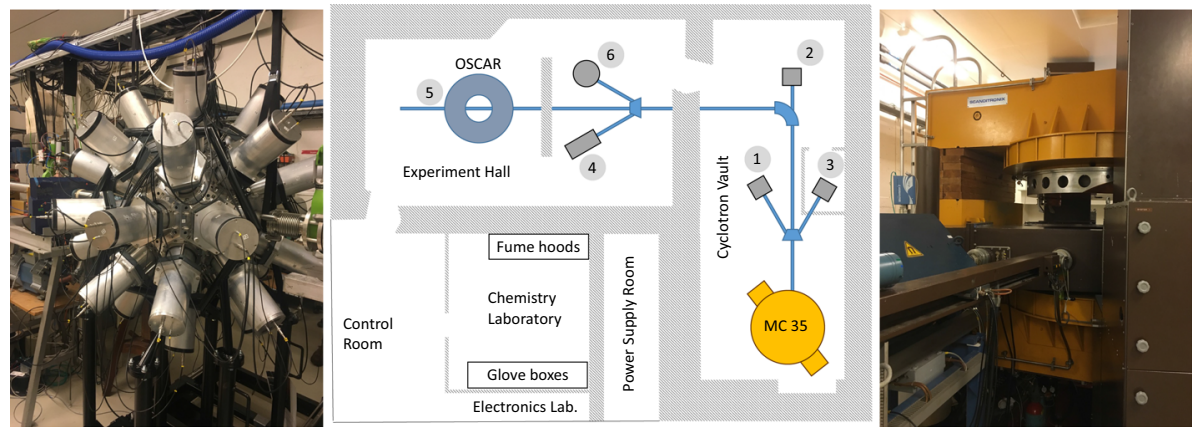

Fig. 1 The OSCAR setup with $30 \mathrm{LaBr}_{3}$ detectors (left). Floor plan of OCL showing the cyclotron vault, the experimental hall, and adjacent laboratories (middle). The beam can be delivered to six different beam lines: (1) solid targets for medical isotope research; (2) high-intensity irradiation of materials in vacuum or air; (3) liquid target station for production of ${ }^{18} \mathrm{~F}$ (presently not in use); (4) irradiation of cells and biological samples in air; (5) OSCAR with auxiliary detectors; (6) scattering chamber with silicon telescope detectors. Photograph of the Scanditronix cyclotron with the yoke jacked up during maintenance (right)

2500 hours per year. Nuclear physics experiments, which typically run continuously over several days, rely on students and academic staff who are trained in the operation of the cyclotron to maintain continuous beam operation. Radiation biology experiments and isotope production runs typically require shorter irradiations and are conducted during day hours with technical staff present. Being a university laboratory, OCL has strong focus on education and produces several master and $\mathrm{PhD}$ theses each year, both at the University of Oslo and at institutions abroad. Construction and operation of experimental equipment also involve strong student participation. Research projects leading to master and $\mathrm{PhD}$ theses are scheduled with priority.

\section{The MC-35 cyclotron and research infrastructure at OCL}

The MC-35 cyclotron was built in 1978 by Scanditronix AB in Uppsala, Sweden. After installation in the basement of the Department of Physics on the Blindern Campus of the University of Oslo, the first beam was extracted in 1979. The cyclotron accelerates ${ }^{1} \mathrm{H}^{+}$ $(8-35 \mathrm{MeV}),{ }^{2} \mathrm{H}^{+}(4-18 \mathrm{MeV}),{ }^{3} \mathrm{He}^{2+}(6-47 \mathrm{MeV})$, and ${ }^{4} \mathrm{He}^{2+}(8-35 \mathrm{MeV})$. The magnet has a pole face diameter of $130 \mathrm{~cm}$ and three sectors with the gap and maximum field strength varying from $10 \mathrm{~cm}$ and $2.0 \mathrm{~T}$ to $18 \mathrm{~cm}$ and $1.3 \mathrm{~T}$. The cyclotron is equipped with eight concentric circular trim coils and three sets of four harmonic coils. The cyclotron has an internal Penning Ionization Gauge (PIG) ion source and is equipped with a recovery system for ${ }^{3} \mathrm{He}$. The acceleration system consists of two dees at $90^{\circ}$ with a maximum accelerating voltage of $40 \mathrm{kV}$. The RF resonators can be tuned between 12 and $27 \mathrm{MHz}$, with a maximum power output of $40 \mathrm{~kW}$. The beam is extracted at a radius of $51 \mathrm{~cm}$ via an electrostatic deflector and a magnetic channel. The beam can be directed to six different target stations, three of which are located in the cyclotron vault itself, and three in a well-shielded experimental hall after passing through a $90^{\circ}$ analyzing magnet. The floor plan of the laboratory is shown in Fig. 1. 
(a)

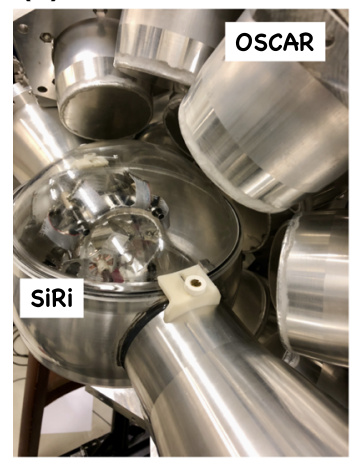

(b)

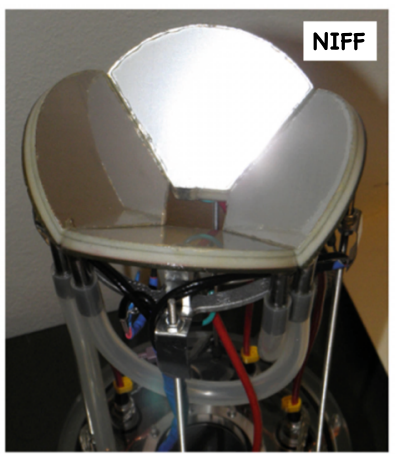

(c)

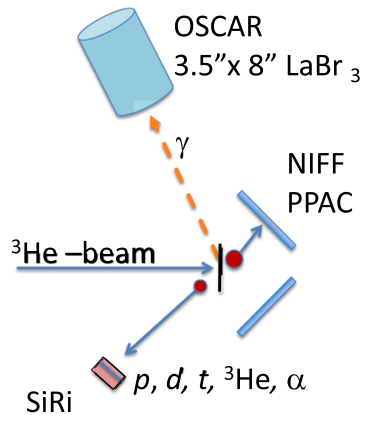

$\Delta \mathrm{E}-\mathrm{E}$ silicon

Fig. 2 a Typical particle- $\gamma$ coincidence setup for the Oslo method. The OSCAR $\mathrm{LaBr}_{3}$ detectors are surrounding the target vacuum chamber with the $64 \mathrm{SiRi}$ particle telescopes. b The NIFF fission detector comprising 4 PPAC. c Schematic drawing (not to scale) of a typical experimental setup comprising the SiRi particle telescopes in backward direction, the NIFF detector for fission fragments in forward direction, surrounded by OSCAR for the detection of $\gamma$ rays

\subsection{Infrastructure for nuclear physics experiments}

Most nuclear physics experiments at OCL rely on the detection of high-energy $\gamma$-rays in coincidence with scattered charged particles. The Oslo Scintillator Array (OSCAR) [1] is a unique $\gamma$-detector system that measures high-energy $\gamma$ rays with high efficiency and good energy resolution under superb timing conditions. Figure 1 shows a picture of the setup, which consists of 30 large-volume $\mathrm{LaBr}_{3}$ :Ce detectors of 3.5 inch diameter and 8 inch length. OSCAR is at present the largest array of $\mathrm{LaBr}_{3}$ detectors in the world. Each detector can be placed in three standard distances of 16,22 , or $25 \mathrm{~cm}$ from the target. The total efficiency is $\approx 15 \%$ for $E_{\gamma}=1.3 \mathrm{MeV}$ with the detectors placed at $22 \mathrm{~cm}$ from the target. OSCAR is replacing the CACTUS array of 285 " $\times 5$ " NaI:Tl detectors, which was operated ac OL until 2018.

Light-ion ejectiles from transfer reactions are detected using the silicon ring (SiRi) detector array [2], see Fig. 2a. The array consists of $64 \Delta E-E$ silicon telescopes arranged in 8 trapezoidal pads in a lampshade geometry. Each front detector has a thickness of $130 \mu \mathrm{m}$ and is segmented into 8 strips covering a constant range of scattering angles. The back detectors have a thickness of $1550 \mu \mathrm{m}$. SiRi has a solid angle coverage of $\sim 6 \%$ and can be mounted either in forward or in backward direction near $45^{\circ}$ or $135^{\circ}$, respectively. For the study of actinides, a fission detector, Nuclear Instrument for Fission Fragments (NIFF) [3], can be mounted inside the target chamber. As shown in Fig. $2 b$, the device consists of four individual parallel-plate avalanche counters (PPAC) covering $60 \%$ of $2 \pi$. The detector has $\sim 100 \%$ intrinsic efficiency for the detection of fission fragments, but is insensitive to light charged particles.

A state-of-the-art data acquisition system has been built to read out energies and time stamps for all detectors. The front-end electronics are based on the CompactPCI/PXI standard and 16-channel Digital Pulse Processors (XIA Pixie-16) for the read-out. The signals from the $\mathrm{LaBr}_{3}$ :Ce detectors of OSCAR are digitized using $500 \mathrm{MHz}$ sampling rate, while the 72 channels of SiRi and other auxiliary detectors (NIFF or HPGe) are sampled at $250 \mathrm{MHz}$ rate. A total of 48 channels at $500 \mathrm{MHz}$ and 96 channels at $250 \mathrm{MHz}$ are available. 
The design, construction, assembly, and commissioning of OSCAR and its associated data acquisition system was to a large extend performed by students and postdoctoral fellows. Student contributions include, for example, GEANT4 simulations during the design phase, assembly, testing, and characterization of individual $\mathrm{LaBr}_{3}: \mathrm{Ce}$ crystals and photomultiplier tubes, and software development for the data acquisition. The support frame and encapsulation of the OSCAR detectors were built by the mechanical workshop at the Department of Physics.

\subsection{Infrastructure for biophysics and medical applications}

Beam line 4 (see Fig. 1) is used for the irradiation of cells and other samples in air. A $50 \mu \mathrm{m}$ thick tungsten window is used to scatter the beam into air, producing a homogeneous particle radiation field covering the whole sample. In addition to the choice of beam parameters, the distance between the sample and beam exit window can be adjusted to choose the desired parameters of the radiation field. A transmission ionization chamber is placed behind the beam exit window to monitor the particle fluence. The fluence measurements are calibrated to the absorbed dose using a Markus plane-parallel ionization chamber. For cell irradiations, the Markus chamber is replaced by a rotating cell dish holder maintained at $37{ }^{\circ} \mathrm{C}(37$ degree Celsius). The linear energy transfer (LET) can be precisely adjusted by the distance from the beam exit window and by inserting thin absorbing layers of plastic. The LET is calculated from Monte Carlo simulations [4]. A simple cell laboratory has been established adjacent to the cyclotron to handle the cells before and after irradiation. Future plans include a setup for animal irradiations. The same beam line is also used for studying the activation of photosensitizers that are used in photodynamic cancer therapy by protons (see Sect. 3.2.1), and for radiation hardness testing of electronics components used in LHC detectors at CERN and for space applications.

\subsection{Infrastructure for radioisotope production and handling}

OCL has a long history of producing radioisotopes both for research and clinical applications. From 1999 until 2006, when the Norwegian Medical Cyclotron Centre was established at the Radium Hospital, OCL has done weekly production runs of ${ }^{18} \mathrm{~F}$ for PET applications. Separate beam lines within the cyclotron vault are available for fitting different target stations that allow irradiation of gas, liquid, and solid target materials (see Fig. 1). Historically, these efforts have led to the characterization and use of target technology to produce a large number of radioisotopes. To achieve higher particle currents for the production of radioisotopes, an internal target is currently being designed.

Adjacent to the cyclotron vault is a class-C radiation safety laboratory, for which an extension and an upgrade to class B are planned. An automated system to transport irradiated targets from the cyclotron vault to the chemistry laboratory is currently being designed. The laboratory is equipped with three fume hoods and two shielded glove boxes, which will be increased to five in the upgraded laboratory. For processing of irradiated targets, there are dedicated boxes for distillation or wet chemistry. Products are analyzed with the help of HPGe detectors, radio high-performance liquid chromatography, and radio thin-layer chromatography. 


\section{Research topics and recent highlights}

\subsection{Nuclear structure and nuclear astrophysics}

The interplay of the microscopic, quantum-mechanical regime, and the macroscopic world is crucial for many physical systems. In particular, average nuclear decay properties and structures at high excitation energies (the quasi-continuum region) are indispensable in a variety of applications such as understanding stellar nucleosynthesis, designing next-generation nuclear reactors, and optimizing transmutation of nuclear waste. The combination of available lightion beams and state-of-the-art detector systems makes OCL an attractive experimental site for such studies. The main focus of nuclear physics research at OCL is on statistical properties of nuclei and their application in nuclear astrophysics.

\subsubsection{The Oslo method and statistical properties of nuclei}

As excitation energy increases, the nuclear levels come so close in energy that conventional discrete spectroscopy is difficult or even impossible to accomplish. In this statistical region, the concepts of well-defined states and the decay rate between them are replaced by average properties such as the nuclear level density (NLD) and $\gamma$-ray strength function $(\gamma \mathrm{SF})$. The Oslo group has developed the so-called Oslo method [5], which has also been adopted to radioactive beam experiments for short-lived nuclei. The method allows for a simultaneous determination of the functional form of the $\operatorname{NLD} \rho$ and the $\gamma \mathrm{SF} f$ in one and the same experiment. The technique is based on a factorization of the decay probability given by $P\left(E_{\gamma}, E\right) \propto E_{\gamma}^{3} f\left(E_{\gamma}\right) \rho\left(E-E_{\gamma}\right)$, where $E$ is the initial excitation energy and $E_{\gamma}$ is the primary $\gamma$ energy. The method is in principle model independent and relies on a set of assumptions that has been thoroughly tested for the statistical decay region [6].

The OSCAR-SiRi combination is ideal for the investigation of statistical decays which require high particle- $\gamma$ coincidence rates. Figure $2 \mathrm{a}$ shows the target chamber with the SiRi particle telescopes, which are used to determine the excitation energy $E_{x}$ of the residual nucleus. Fission fragments can be measured with the NIFF detector of Fig. 2b. An illustration of a full setup is shown in Fig. 2c for the coincidences between one charged ejectile, one fission fragment, and one or more $\gamma$ rays.

The nuclear many-particle system represents a perfect microcanonical ensemble, totally isolated with sharp excitation energy $E$ and particle numbers $N$ and $Z$. The density of levels as a function of excitation energy is the starting point to extract thermodynamic quantities such as entropy, temperature, and heat capacity. Provided that the level density $\rho(E)$ is proportional to the number of accessible states, we may express the multiplicity of states as $\Omega(E) \propto \rho(E)$. This leads to the most important quantity in thermodynamics, namely entropy $S(E)=k_{B} \ln \Omega(E)+S_{0}$, where $S_{0}$ is a constant and $k_{B}$ is the Boltzmann constant. The corresponding microcanonical temperature and heat capacities are given by $T(E)=$ $(\partial S / \partial E)^{-1}$ and $C_{V}(E)=(\partial T / \partial E)^{-1}$.

Figure 3 shows how these quantities can be extracted by the Oslo method using the example of the ${ }^{237,238,239} \mathrm{U}$ isotopes [7]. The almost linear entropy reveals that the NLD follows a close-to-constant temperature model characterized by a critical temperature of $T_{C} \approx 0.4 \mathrm{MeV}$. This indicates that heating the nuclear system results in breaking of Cooper pairs rather than an increase in temperature. These phenomena are in close analogy with the process of melting ice. Furthermore, from the odd-even mass difference in entropy, we observe that each quasi-particle carries an entropy of $\Delta S \approx 1.7 k_{B}$. 
(a)

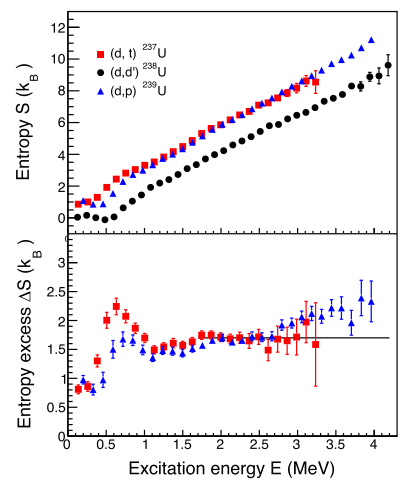

(b)

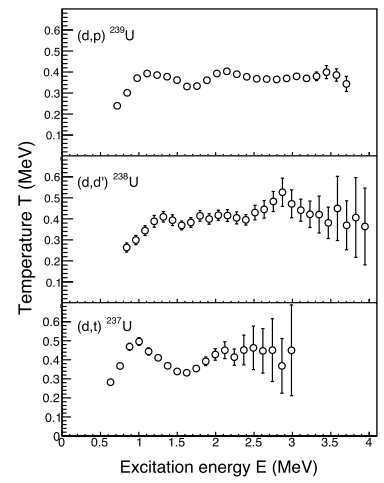

(c)

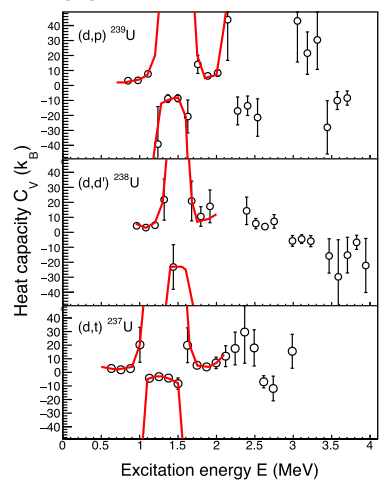

Fig. 3 a Thermodynamic properties in the microcanonical ensemble obtained for ${ }^{237,238,239} \mathrm{U}$. The level density curves are exponentially increasing functions that suggest a linear entropy as function of excitation energy. The entropy difference between odd-mass and even-even isotopes is $\Delta S \approx 1.7 \mathrm{k}$. b From the entropy, the nuclear temperature is found to be $T \approx 0.4 \mathrm{MeV}$. c The deduced heat capacities go asymptotically at the critical excitation energies $E \approx 1.0-1.5 \mathrm{MeV}$. The data have been previously published [7]

(a) ${ }^{56} \mathrm{Fe}\left(p, p^{\prime} \gamma\right)^{56} \mathrm{Fe}$

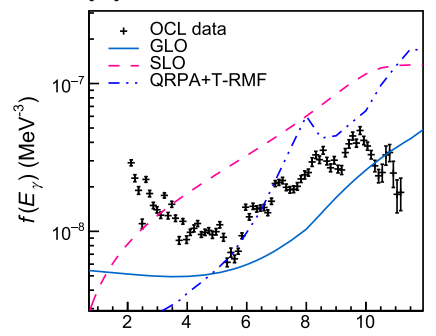

(b) ${ }^{232} \mathrm{Th}(d, p \gamma)^{233} \mathrm{Th}$

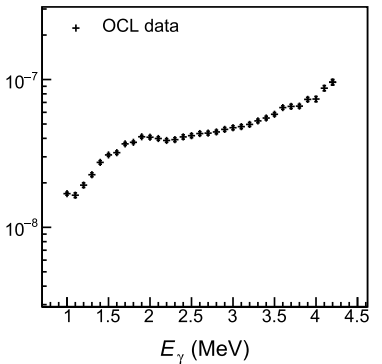

(c) ${ }^{117} \mathrm{Sn}\left({ }^{3} \mathrm{He},{ }^{3} \mathrm{He} \cdot \mathrm{y}\right)^{17} \mathrm{Sn}$

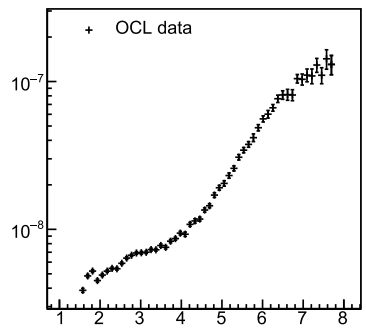

Fig. 4 Gamma-ray strength functions of $\mathbf{a}^{56} \mathrm{Fe}$ [8], $\mathbf{b}^{233} \mathrm{Th}$ [9], and $\mathbf{c}^{117} \mathrm{Sn}$ [10]. Note that the range of the $\mathrm{y}$ axis is kept fixed, while the range of the $\mathrm{x}$ axis varies. In the case of ${ }^{56} \mathrm{Fe}$, the measured $\gamma \mathrm{SF}$ is compared to commonly used models available in the nuclear reaction code TALYS-1.8 [12]

The study of the $\gamma \mathrm{SF}$ in the quasi-continuum has also been very fruitful. Figure 4 shows three examples of $\gamma \mathrm{SFs}$ measured at OCL. One of the most surprising observations was that the $\gamma \mathrm{SF}$ starts to increase for decreasing $\gamma$ energies below a few $\mathrm{MeV}$ for many light nuclei. This low-energy enhancement, which is often called the $\gamma \mathrm{SF}$ up-bend, is evident for ${ }^{56} \mathrm{Fe}$ in Fig. 4a [8]. Also the scissors resonance (SR) embedded in the quasi-continuum, e.g., for ${ }^{233} \mathrm{Th}$ in Fig. 4b [9], has been systematically studied by the Oslo group. Finally, at slightly higher $\gamma$-ray energies, the so-called pygmy dipole resonance (PDR) can be observed as enhanced $\gamma$ decay in the quasi-continuum, e.g., in the tin and gold mass regions, see Fig. 4c [10]. All these structures, which are superimposed on the tail of the Giant Dipole Resonance (GDR), enhance the probability for ( $n, \gamma$ ) capture reactions, and are therefore important for astrophysical reaction rates, as will be discussed below. With a large amount of data obtained for a wide range of nuclei, OCL is a major contributor to the IAEA reference database for photon strength functions [11]. 


\subsubsection{Nuclear astrophysics: heavy-element nucleosynthesis}

Two neutron-capture processes, named the slow $(s)$ and rapid $(r)$ neutron-capture process according to their vastly different time scales, have each produced roughly half of the heavy-element isotopes observed in the solar system, while the $p$ process (proton capture/photodisintegration) is responsible for about 35 nuclides not created in the $s$ and $r$ processes. In addition to these "classical" nucleosynthesis processes, an intermediate $(i)$ neutron-capture process might also play a role in certain stellar environments.

To be able to understand the heavy-element nucleosynthesis processes and to explain the element abundances, large nuclear reaction networks are applied. A crucial input to the networks is the various creation and destruction probabilities (astrophysical reaction and decay rates) for each nucleus included in the network. As both the $i$ and $r$ processes involve (very) neutron-rich nuclei, there is little to no experimental information on these rates. Even for the $s$ process, which mostly follows the valley of stability, there are $s$-process branchings for which no experimental data exist on radiative neutron-capture rates. Moreover, chargedparticle reactions such as $(p, \gamma)$ and $(\alpha, \gamma)$ for the $p$-process reaction network are very difficult to measure directly for sub-Coulomb energies. When there is no data available, one relies on theoretical estimates on the reaction rates, which can diverge by orders of magnitude.

One way to provide experimental constraints on the astrophysical rates is to measure the NLD and $\gamma \mathrm{SF}$ of the residual nucleus in the radiative capture process, be it $(n, \gamma)$ for the $s$, $i$, and $r$ processes or $(p, \gamma)$ and $(\alpha, \gamma)$ for the $p$ process. Using these experimental NLDs and $\gamma$ SFs as input to nuclear reaction codes, one obtains a significantly improved prediction of the radiative capture rate. The Oslo method described in Sect. 3.1.1 is a unique tool to achieve experimental NLDs and $\gamma$ SFs simultaneously from a data set of charged-particle- $\gamma$ coincidences. The data provide stringent tests on theoretical calculations of both quantities, as well as playing a big role in determining radiative cross sections and reaction rates.

One example of a data-constrained cross section for the $p$ process is the case of ${ }^{91} \mathrm{Nb}(p, \gamma){ }^{92} \mathrm{Mo}$, which is of importance to the puzzling ${ }^{92}$ Mo abundance [13]. Tveten et al. [14] provided a first experimentally constrained ${ }^{91} \mathrm{Nb}(p, \gamma)^{92}$ Mo reaction rate by measuring the NLD and $\gamma \mathrm{SF}$ of ${ }^{92} \mathrm{Mo}$, and concluded that the ${ }^{92}$ Mo underproduction is not due to the nuclear-physics input. Moreover, a benchmark of our approach was obtained for the

${ }^{88} \operatorname{Sr}(p, \gamma){ }^{89} \mathrm{Y}$ cross section [15], see Fig. 5a. For $(n, \gamma)$ cross sections, the ${ }^{161} \operatorname{Dy}(n, \gamma)$ case served as an excellent benchmark as there were many measurements available, see Fig. $5 \mathrm{~b}$. Using the data of Renstrøm et al. [16], a very good agreement with the direct $(n, \gamma)$ data was found. Furthermore, for the $s$-process branching at the unstable ${ }^{191}$ Os, Kullmann et al. [17] provided experimental support to the adopted value of the KADoNiS database [18]. In the near future, $(\alpha, p \gamma)$ reactions will be applied to access moderately neutron-rich nuclei of great importance for the $i$ process, while at the same time bridging the gap towards $r$-process nuclei that will be studied at large radioactive-beam facilities.

\subsubsection{Recent highlight: radiative width of the Hoyle state}

The combination of light-ion beams and efficient detector arrays for particle and $\gamma$-ray detection is not only ideal for measurements in the quasi-continuum, as described above, but also to study rare decay processes using discrete spectroscopy. This was recently demonstrated by a study of the radiative width of the Hoyle state in ${ }^{12} \mathrm{C}$ [19]. The existence of a resonant state in ${ }^{12} \mathrm{C}$ was predicted by Hoyle in 1953 as a steppingstone to explain the production of carbon, and hence all elements beyond, through the triple- $\alpha$ process. An excited $0^{+}$state at the predicted excitation energy of $7.65 \mathrm{MeV}$, termed the Hoyle state, was discovered soon 
(a) ${ }^{88} \operatorname{Sr}(p, y)$

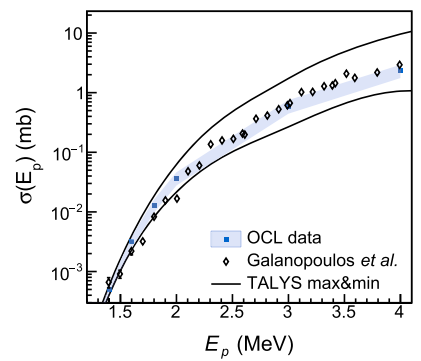

(b) ${ }^{161} \mathrm{D} y(n, \gamma)$

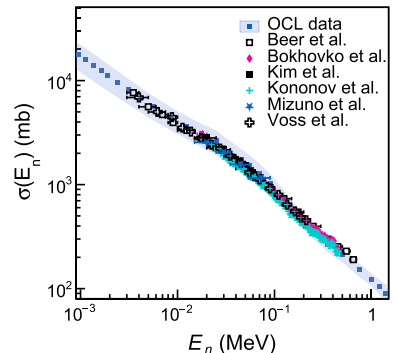

(c) ${ }^{191} \mathrm{Os}(\mathrm{n}, \gamma)$

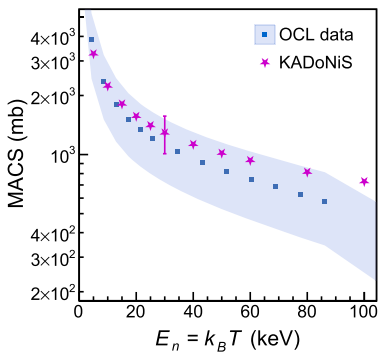

Fig. 5 a Deduced ${ }^{88} \operatorname{Sr}(p, \gamma){ }^{89} \mathrm{Y}$ cross section from ${ }^{89} \mathrm{Y}\left(p, p^{\prime} \gamma\right)$ data measured at OCL [15] compared to a direct measurement. Also shown are the minimum and maximum predicted cross sections using TALYS-1.6 (modified from Ref. [15]). b Comparison of the experimentally constrained ${ }^{161} \operatorname{Dy}(n, \gamma)$ cross section using OCL data [16] to direct measurements (modified from Ref. [16]). c Deduced ( $n, \gamma)$ Maxwellian-averaged cross section (MACS) of the $s$-process branch-point nucleus ${ }^{191}$ Os from ${ }^{192} \operatorname{Os}\left(\alpha, \alpha^{\prime} \gamma\right)$ data measured at OCL [17], compared to the KADoNiS recommended MACS [18] (modified from Ref. [17])

afterward, and has been studied in great detail since (see, e.g., Ref. [20]). The radiative width of the state, i.e., the probability to decay to the stable ground state in ${ }^{12} \mathrm{C}$ instead of breaking apart into three $\alpha$ particles again, was measured in several experiments during the 1960 s and 70 s, and was found to be small with approximately 1 in 2500 carbon atoms surviving the triple- $\alpha$ process. A new experiment at OCL aimed at measuring the $\gamma$-ray cascade from the Hoyle state and improve the uncertainty for the radiative width. The experiment, which was a collaboration between scientists from the Australian National University and the University of Oslo, used inelastic proton scattering on ${ }^{12} \mathrm{C}$ to populate the state. Events in which the Hoyle state was populated were identified by the energy of the scattered proton detected in $\mathrm{SiRi}$, while $\gamma$ rays were detected with the previous detector array CACTUS. During a weeklong experiment, a total of $2.78(6) \times 10^{8}$ Hoyle state events were recorded. Out of these, only $217(21)$ decayed by a $\gamma$-ray cascade via the $2_{1}^{+}$state to the ground state of ${ }^{12} \mathrm{C}$. This result yields a radiative width of $\Gamma_{\text {rad }}=5.1(6) \times 10^{-3} \mathrm{eV}$. This value is about $34 \%$ higher than the currently adopted value and will impact models of stellar evolution and nucleosynthesis. Details on these findings can be found in Ref. [19]. Because of the deviation from previous measurements and the potential high impact on nucleosynthesis models, confirmation of the result is needed. New experiments with OSCAR are already under way, which can be expected to yield even better accuracy due to the efficiency and resolution of the $\mathrm{LaBr}_{3}: \mathrm{Ce}$ detectors.

\subsection{Medical physics and radiation biology}

For some decades now, accelerated-proton radiotherapy has been gaining ground in the anticancer arena, since protons can penetrate deep into the tissue and also deliver highenergy payloads at the cancer site selectively in the energy region of the so-called Bragg peak. Nevertheless, proton therapy, as other radiotherapies, may trigger side effects and also runs the risks of mutagenesis and carcinogenesis. Despite the extensive application of radiotherapy in cancer treatment, the physical and biological mechanisms underlying its therapeutic effects are not fully understood. The implementation of radiotherapy is to a large extend based on clinical experience with X-ray irradiation, with the absorbed dose being the most important parameter. However, protons and X-rays deposit energy by different processes and produce ion clusters of different size and density, which affects the nature 


\section{(a)}

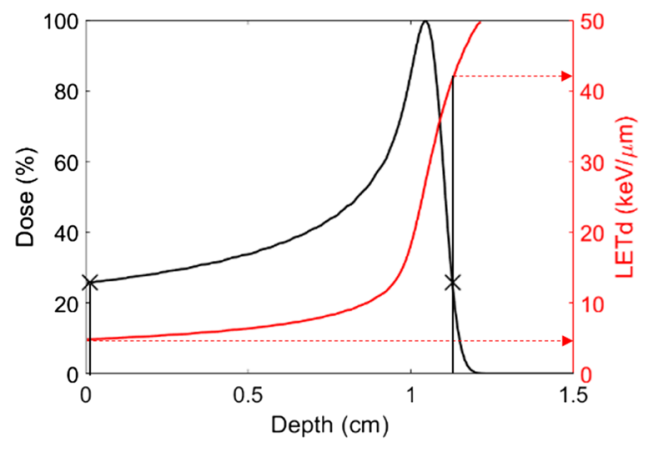

(b)

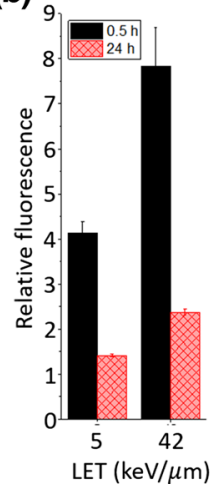

(c)

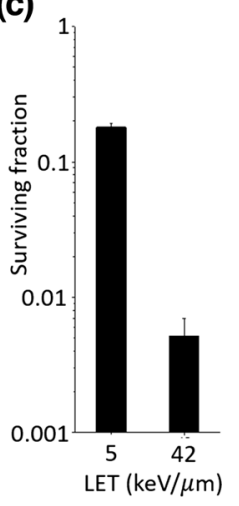

Fig. 6 a Monte Carlo simulated depth dose curve (black line) and dose-averaged LET (red line). The two positions (LET of $5 \pm 0.6$ and $42 \pm 2 \mathrm{keV} / \mu \mathrm{m}$ ) used in panels B and C are marked [4]. b Amount of DNA double-strand breaks as a function of LET. T98G glioblastoma cells were irradiated with 5 Gy in two different positions. The relative values of $\gamma-\mathrm{H} 2 \mathrm{AX}$ fluorescence intensity were used as marker for DNA double-strand breaks. The values were normalized to the levels for unirradiated T98G cells. The cells were harvested $0.5 \mathrm{~h}$ (initial DNA damage) or $24 \mathrm{~h}$ (residual damage after repair) after irradiation. c Surviving fraction as a function of LET. The cell survival was measured by the clonogenic assay

and complexity of damages to macromolecules such as DNA. The physical, chemical, and biological processes in biological tissue will therefore be different after interaction with the same dose of protons or X-rays, or of the same type of radiation given with different timing or energy. The research program in medical physics and radiation biology at OCL aims at obtaining better comprehension of the physical and biological mechanisms underlying proton therapy. The program is well aligned with the establishment of a proton therapy center at the Oslo University Hospital in 2023.

More specifically, experiments at OCL investigate how cancer cells respond to proton radiation in terms of reduced survival, DNA damage, cell signaling, and importance for immune response. With the precise dosimetry setup described in Sect. 2.2, it is possible to irradiate cancer cells and study their biological response to varying linear energy transfer (LET) along the proton track and, in particular, at the end of the Bragg peak. For this purpose, the energy range of protons available at OCL is ideal. Applying the same dose at different positions in the proton track, we have demonstrated a much higher number of DNA doublestrand breaks at the end of Bragg peak [21]. The unrepaired number of double-strand breaks after $24 \mathrm{~h}$ is highly correlated with cellular surviving fraction (measured by the colony assay) for varying LETs. Examples for such measurements are presented in Fig. 6a-c, where T98G glioblastoma cells were irradiated with the same dose of $5 \mathrm{~Gy}$ in two different positions of the Bragg peak, corresponding to different LET. The relative values of $\gamma-\mathrm{H} 2 \mathrm{AX}$ fluorescence intensity were used as marker for DNA double-strand breaks. As can be seen from Fig. 6, cell survival per dose is much lower at the end of the Bragg peak. We are presently investigating whether this LET-dependent induction of cell death reflects a higher immunogenicity in the cells. The hypothesis is that proton irradiation induces immunogenic cell death, stimulating emission of signal to induce an anti-tumor immune response [22-25]. This may open for new combinatory cancer treatments with immune therapy. 


\subsubsection{Recent highlight: proton-induced activation of photosensitizers}

In contemporary photomedicine, photodynamic therapy of cancer (PDT) is a major therapeutic modality against solid tumors [26,27]. In PDT, a photosensitive drug (photosensitizer) absorbs visible light of an appropriate wavelength, and during the transitions to its excited state, it interacts with the ambient molecular oxygen to create reactive oxygen species, predominantly singlet oxygen [28]. These reactive oxygen species kill the cancer cells, to which the light is selectively directed. Photosensitizers have also been known to selectively accumulate in cancerous lesions, like the incurable brain cancer glioblastoma multiforme (GBM), with a high selectivity, thus giving the ability to surgeons to accurately resect the GBM tumors guided by the fluorescence of the photosensitizer [29,30]. This selective accumulation of photosensitizers in lesions like GBM could be highly beneficial for PDT [31]. However, light from an external source such as a lamp or a laser cannot penetrate deep enough into the tissue to reach inner tumors, making the whole operation invasive.

Standard proton radiotherapy utilizes the ionization caused by the protons along their path. Possible effects of electron excitations, however, have largely been overlooked so far. A series of experiments at OCL addressed the question whether photosensitizers can be activated by the passing protons and consequently be used as an additional treatment of the malignancies. A beam of $16 \mathrm{MeV}$ protons was used to irradiate a library of photosensitizers and study their excitation. The proof of this excitation came through the registration of proton-induced fluorescence with the use of a spectrograph, but also with a conventional photographic camera. The image presented in Fig. 7c shows the fluorescence of a photosensitizer irradiated by protons. It was further demonstrated that protons are able to drive photosensitizers into their triplet states, where they can interact with molecular oxygen, through their phosphorescence in dry gels. We also documented the generation of singlet oxygen: i) directly using a nearinfrared photomultiplier tube to register the singlet oxygen phosphorescent decay to its ground triplet state at $1270 \mathrm{~nm}$; and ii) indirectly through the generation of singlet-oxygen-related photosensitizer photoproducts, which could be quenched by the addition of singlet oxygen scavengers. Finally, the proof of principle of proton dynamic therapy was obtained in GBM cells, treated with the naturally occurring photosensitizer cercosporin, where more than $20 \%$ average cell death was achieved in addition to that of the radiotherapy. Examples from the cell survival study are shown in Fig. 7a and b. Further details of the experimental work and its results can be found in Ref. [32]. The results obtained at OCL are expected to pave the way for a hybrid therapy combining proton radiotherapy and proton-dynamic therapy, readily applicable in all future and existing clinical proton therapy centers.

\subsection{Research and development on medical isotopes}

Since the beginning of its operation in 1979, numerous projects on radionuclide production for industrial and clinical use have been conducted at OCL within an interdisciplinary research environment between radiochemistry and nuclear physics. Although OCL has produced ${ }^{18} \mathrm{~F}$ for clinical PET application over a longer period, the focus has always been on research and development rather than production. With a range of available beam particles and energies going beyond what is typically available at hospital cyclotrons, a focus has been on $\alpha$ emitters such as ${ }^{211} \mathrm{At}$, which requires $\alpha$ beams of $\sim 30 \mathrm{MeV}$ to drive the reaction ${ }^{209} \mathrm{Bi}(\alpha, 2 n){ }^{211} \mathrm{At}$ [33]. Due to decreasing availability of nuclear reactors and increasing demand, the manufacturing of radionuclides for medical and industrial applications is shifting towards the use of particle accelerators for production. At the same time, there has been tremendous progress in utilizing the specific physical and chemical properties of a much wider range 

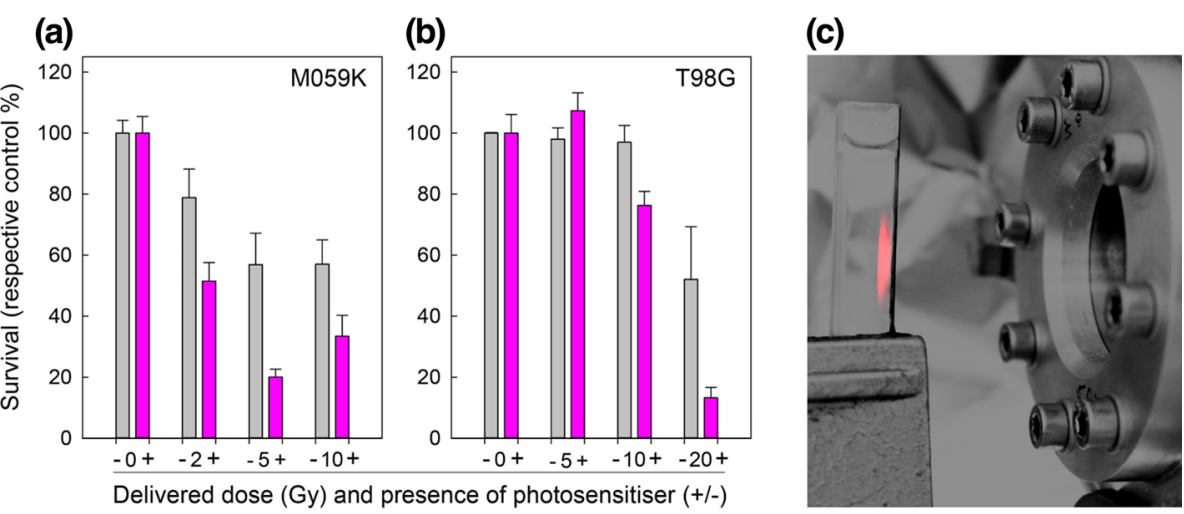

Fig. 7 Proof of principle of proton dynamic activation of the photosensitizer cercosporin on the human glioblastoma cell lines, M059K (a) and T98G (b). Cells were irradiated with 0 (non-irradiated controls), 2, 5, 10 , and $20 \mathrm{~Gy}$ with/without cercosporin incubation (4-10 $\mu \mathrm{M}, 4 \mathrm{~h})$. Gray bars represent cell survival in cells not treated with photosensitizer, while magenta bars denote survival of cercosporin-treated cells. Errors are represented as standard errors of the mean. The figure is reproduced from Ref. [32]. Panel c shows the protoninduced fluorescence from a photosensitizer solution. Real-time-proton-induced fluorescence is overlapped with a photograph of the cuvette. The beam exit window can be seen on the right

of radioisotopes than those traditionally used in medical applications. This brings along a need to develop production technology for both dedicated accelerator facilities and existing hospital cyclotrons.

The present research program at OCL is mostly focused on developing target technology that withstands the heat deposition from high beam currents. Today, the production yield is in most cases limited by the beam current that a target can withstand. We aim to overcome these physical challenges by utilizing the chemical properties of thermostable materials and develop dedicated post-irradiation processing capabilities. We have developed a strategy for making production targets from phosphate-based materials, such as ceramic and glass, which can withstand high temperatures and have favorable properties for final chemical processing. The glass matrix can physically and chemically immobilize and house target elements in high content, up to approximately $40 \%$ by weight. It is a further advantage that these materials can be formed in any geometry to fit different target holders before irradiation. In the design of protocols for the workup of products, we aim for processing steps that can be implemented with automated equipment for remote handling.

\subsection{Testing of scintillating materials for ESS}

The European Spallation Source (ESS), currently under construction in Lund, Sweden, will be the most powerful neutron source in the world [34]. The driver of the neutron production is a $5 \mathrm{MW}$ proton beam hitting a rotating five tonne tungsten target wheel. The proton beam line will have several diagnostic systems, including four beam imaging systems. The beam imaging systems consist of scintillating material that converts some of the proton beam energy into light, an optical path consisting of mirrors to transport the light to an area where cameras can be operated, and electronics and signal processing to extract information from the images. Two of the imaging systems will be installed near the tungsten target, with two further systems along the tuning dump line. The imaging systems are being developed by the University of Oslo as an in-kind contribution [35,36]. 
Scintillating materials have been in use for beam imaging at accelerators for decades, but the performance of scintillators with the amount of radiation present at the ESS target needs to be assessed. The Spallation Neutron Source at Oak Ridge National Laboratory has used $\mathrm{Cr}: \mathrm{Al}_{2} \mathrm{O}_{3}$ that is coated on metal surfaces as a scintillator for imaging systems. ESS will coat the target wheel, as well as the proton beam window separating the accelerator from the target area, with the same material. Coating makes it possible to add very thin layers of scintillator to structures that are already cooled. The OCL is being used as a part of the qualification of scintillator coatings for the ESS target imaging systems. Samples of coated aluminum are mounted on an in-air Faraday cup for measuring the beam current. Images and optical spectra are recorded, and correlated with the Faraday cup readings. A heating gun is also available for measuring light yield and spectra as a function of temperature. The coating process affects both the amount of doping and the crystal structure of the coating, which are both linked to performance. The goal of the qualification is to select the best process for applying $\mathrm{Cr}: \mathrm{Al}_{2} \mathrm{O}_{3}$ for the initial target systems, but also to look for alternative chemical compositions for upgrades in the future.

\section{Summary and outlook}

The Oslo Cyclotron Laboratory is a small-scale, university-based accelerator laboratory with a diverse scientific program ranging from nuclear structure and nuclear astrophysics to radiation biology and medical applications. The nuclear physics group at the Department of Physics has over many years developed the infrastructure and methodology to study statistical properties of highly excited nuclei in the quasi-continuum region, and gained a leading position in this specific sub-field of nuclear physics research. Experiments using the Oslo Method provide important data for nuclear astrophysics and nuclear energy applications. The new OSCAR detector array is a unique instrument which provides new opportunities for experiments that have not been possible before, widening the scope of nuclear physics research at OCL. The biophysics and medical physics group at the Department of Physics has, together with researchers from the University Hospital, established a fruitful program in radiation biology at OCL. Radiochemistry and medical isotope research have been a focus of activity at OCL for many years. Upgrades of the cyclotron itself and the associated radiochemistry laboratory are expected to boost this activity further. The laboratory has a broad user base within the University of Oslo, with active collaborators from around the world. As a university laboratory, OCL has a strong focus on educating master and $\mathrm{PhD}$ students, who have the opportunity to gain hands-on experience in experimental work with state-of-the-art equipment.

Funding Open Access funding provided by University of Oslo (incl Oslo University Hospital).

Open Access This article is licensed under a Creative Commons Attribution 4.0 International License, which permits use, sharing, adaptation, distribution and reproduction in any medium or format, as long as you give appropriate credit to the original author(s) and the source, provide a link to the Creative Commons licence, and indicate if changes were made. The images or other third party material in this article are included in the article's Creative Commons licence, unless indicated otherwise in a credit line to the material. If material is not included in the article's Creative Commons licence and your intended use is not permitted by statutory regulation or exceeds the permitted use, you will need to obtain permission directly from the copyright holder. To view a copy of this licence, visit http://creativecommons.org/licenses/by/4.0/. 


\section{References}

1. F. Zeiser et al., Nucl. Instr. Methods A 985, 164678 (2021)

2. M. Guttormsen et al., Nucl. Instr. Methods A 648, 168 (2011)

3. T.G. Tornyi et al., Nucl. Instr. Methods A 738, 6 (2014)

4. T.J. Dahle et al., Acta Oncologica 56, 779 (2017)

5. A. Schiller et al., Nucl. Instr. Methods A 447, 498 (2000)

6. A.C. Larsen et al., Phys. Rev. C 83, 034315 (2011)

7. M. Guttormsen et al., Phys. Rev. C 88, 024307 (2013)

8. A.C. Larsen et al., Phys. Rev. Lett. 111, 242504 (2013)

9. M. Guttormsen et al., Phys. Rev. Lett. 109, 162503 (2012)

10. U. Agvaanluvsan et al., Phys. Rev. Lett. 102, 162504 (2009)

11. S. Goriely et al., Eur. Phys. J. A 55, 172 (2019)

12. A. J. Koning, S. Hilaire, and M.C. Duijvestijn, in Proceedings of the International Conference on Nuclear Data for Science and Technology 2007, ed. O. Bersillo et al., (EDP Sciences, Les Ulis, 2008), p. 211

13. T. Rauscher et al., Rep. Prog. Phys. 76, 066201 (2013)

14. G.M. Tveten et al., Phys. Rev. C 94, 025804 (2016)

15. A.C. Larsen et al., Phys. Rev. C 93, 045810 (2016)

16. T. Renstrøm et al., Phys. Rev. C 98, 054310 (2018)

17. I.K.B. Kullmann et al., Phys. Rev. C 99, 065806 (2019)

18. "KADoNiS v0.3" - The third update of the "Karlsruhe Astrophysical Database of Nucleosynthesis in Stars"; I. Dillmann, R. Plag, F. Käppeler, T. Rauscher, https://exp-astro.de/kadonis0.3/

19. T. Kibédi et al., Phys. Rev. Lett. 125, 182701 (2020)

20. M. Freer, H.O.U. Fynbo, Prog. Part. Nucl. Phys. 78, 1 (2014)

21. N.F.J. Edin et al., Radiotherapy Oncol. 133, 594 (2019)

22. A.B. Sharabi et al., Lancet Oncol. 16, e498 (2015)

23. Y. Huang et al., Ann. Transl. Med. 7, 542 (2019)

24. S. Kumari et al., Int. J. Mol. Sci. 21, 8151 (2020)

25. S.R. Gameiro et al., Int. J. Radiat. Oncol. Biol. Phys. 95, 120 (2016)

26. A..J. MacRobert, T. Theodossiou, in Encyclopedia of Modern Optics, ed. by R.. D. Guenther (Elsevier, Amsterdam, 2005), p. 53

27. P. Agostinis et al., CA Cancer J. Clin. 61, 250 (2011)

28. B. Li et al., J. Biophoton. 9, 1314 (2016)

29. W. Stummer et al., Neurosurgery $\mathbf{4 2 ,} 518$ (1998)

30. W. Stummer et al., J. Neurosurg. 93, 1003 (2000)

31. M.-C. Tetard et al., Photodiagnosis Photodyn. Therapy 11, 319 (2014)

32. M. Grigalavicius et al., Nat. Commun. 10, 3986 (2019)

33. G. Henriksen et al., Appl. Radiat. Isot. 54, 839 (2001)

34. R. Garoby et al., Phys. Scr. 93, 014001 (2018)

35. E. Adli et al., Proc. of International Particle Accelerator Conference (IPAC'17), Copenhagen, p. 3422 (2017)

36. M.G. Ibison et al., Nucl. Instr. Methods A 950, 162790 (2020) 